\section{ORIGINAL RESEARCH}

Z. Kulcsár

Á. Ugron

M. Marosfő $i$

Z. Berentei

G. Paál

I. Szikora

\title{
Hemodynamics of Cerebral Aneurysm Initiation: The Role of Wall Shear Stress and Spatial Wall Shear Stress Gradient
}

BACKGROUND AND PURPOSE: Cerebral aneurysms are preferentially located at arterial curvatures and bifurcations that are exposed to major hemodynamic forces, increasingly implicated in the life cycle of aneurysms. By observing the natural history of aneurysm formation from its preaneurysm state, we aimed to examine the hemodynamic microenvironment related to aneurysm initiation at certain arterial segments later developing an aneurysm.

MATERIALS AND METHODS: The 3 patients included in the study underwent cerebral angiography with 3D reconstruction before a true aneurysm developed. The arterial geometries obtained from the 3D-DSA models were used for flow simulation by using finite-volume modeling. The WSS and SWSSG at the site of the future aneurysm and the flow characteristics of the developed aneurysms were analyzed.

RESULTS: The analyzed regions of interest demonstrated significantly increased WSS, accompanied by an increased positive SWSSG in the adjacent proximal region. The WSS reached values of $>5$ times the temporal average values of the parent vessel, whereas the SWSSG approximated or exceeded peaks of $40 \mathrm{~Pa} / \mathrm{mm}$ in all 3 cases. All patients developed an aneurysm within 2 years, 1 of which ruptured.

CoNCLUSIONS: The results of this hemodynamic study, in accordance with the clinical follow-up, suggest that the combination of high WSS and high positive SWSSG focused on a small segment of the arterial wall may have a role in the initiation process of aneurysm formation.

ABBREVIATIONS: AcomA = anterior communicating artery; $\mathrm{AFI}=$ aneurysm formation indicator; $\mathrm{CFD}=$ computational fluid dynamics; DSA = digital subtraction angiography; $\mathrm{GON}=$ gradient oscillatory number; ICA = internal carotid artery; MRA = MR angiography; PcomA = posterior communicating artery; SWSSG = spatial wall shear stress gradient; WSS = wall shear stress: WSSg $=$ wall shear stress gradient

D uring the past, intensive research has been focused on the role of hemodynamic forces in arterial pathologies. Cerebral aneurysms are preferentially located at the outer curvatures, bifurcations, or branching points of the brain vessels. As shown by CFD modeling, these vascular segments are affected by complex hemodynamic forces, including impinging flow and alteration of the WSS, ${ }^{1-3}$ which may induce pathologic remodeling of the vascular structure, transmitted by the endothelial cells. ${ }^{4-6}$

The hemodynamic microenvironment that may be triggering the initiation of aneurysm formation has not yet

Received May 7, 2010; accepted after revision August 12.

From the Department of Interventional Neuroradiology (Z.K., M.M., Z.B., I.S.), National Neuroscience Institute, Budapest, Hungary; Department of Neuroradiology (Z.K.), Hirslanden Clinic, Zurich, Switzerland; and Department of Hydrodynamic Systems (G.P.), Budapest University of Technology and Economics, Budapest, Hungary.

This work was partially supported by a grant from the Hungarian National Scientific and Research Fund (OTKA 73773), and by the "Öveges József" PhD scholarship of GE Hungary ZRt Healthcare Division. This work is also related to the scientific program of the "Development of Quality-Oriented and Harmonized $\mathrm{R}+\mathrm{D}+\mathrm{I}$ Strategy and Functional Model at the BUTE" project. That project is supported by the New Hungary Development Plan (project ID: TÁMOP-4.2.1/B-09/1/KMR-2010-0002).

Please address correspondence to István Szikora, MD, PhD, Department of Interventional Neuroradiology, National Neuroscience Institute, Budapest, Hungary, Amerikai ut 57. Budapest 1145, Hungary; e-mail: h13424szi@ella.hu

Indicates open access to non-subscribers at www.ajnr.org

DOI 10.3174/ajnr.A2339

been clearly elucidated. Animal studies on artificially created vessel bifurcations, combined with CFD simulations, have suggested the coexistence of increased WSS and positive SWSSG at the bifurcation as triggers of aneurysm initiation, and this was supported by histologic findings. ${ }^{7,8}$ CFD studies on real human vessels harboring a sidewall aneurysm were also performed after virtual removal of the aneurysm, focusing on the affected vascular segment at its assumed "healthy" state. ${ }^{9,10}$ Mantha et al ${ }^{9}$ suggested that stagnation zones, with an area of relatively low and rotating WSSs coincide with aneurysm-formation locations, and they introduced the AFI index for the detection of these zones. However, Shimogonya et $\mathrm{al}^{10}$ could not reproduce the correlation of either the AFI or the SWSSG with the location of aneurysm formation, and they introduced the GON for this purpose, which represents the fluctuation of SWSSG integrated over 1 pulse cycle. In these studies, only sidewall aneurysms were included, and the vascular geometry was artificially reconstructed to its assumed preaneurysmal state, insinuating that vessel wall remodeling does not take place during the process of aneurysm development and growth.

In observing the natural history of aneurysm formation from its preaneurysm state, our purpose was to examine the hemodynamic microenvironment related to aneurysm initiation at certain arterial segments later developing an aneurysm. The available 3D-DSA images, representing the true situation 
before aneurysm initiation at both sidewall and bifurcation locations, provided a basis for CFD simulations.

\section{Materials and Methods}

Three patients who were scanned from the introduction of 3D-DSA imaging at our institution and for 9 years after were selected for analysis. During the follow-up, these patients developed a saccular aneurysm at a location where, at the time of the initial angiogram including 3D-DSA, no aneurysm was present (2 patients) or only a small aneurysm-like dilation was demonstrated (1 patient). The studies were obtained due to ruptured (2 cases) and incidentally discovered aneurysms ( 1 case) at different locations from the studied segments. Medical history, risk factors, and the time between the initial angiogram and the follow-up studies showing a developed aneurysm were recorded for all patients.

CFD modeling was performed in all 3 patients at baseline and also in 2 patients after aneurysm development. The CFD modeling was performed similarly, as previously described. ${ }^{11,12}$ Briefly, the initial 3D rotational angiography obtained by a LCV + DSA unit (GE Healthcare, Chalfont St. Giles, United Kingdom) was reconstructed by using an Advantage Windows workstation with ADW 4.2 reconstruction software (GE Healthcare, Milwaukee, Wisconsin). The 3D image data base was then converted to a 3D surface. The commercially available ICEM CFD 11.0 (ANSYS, Canonsburg, Pennsylvania) was used to generate an advanced tetrahedral mesh inside the extracted surface. To have a good numeric resolution near the vessel wall, we used 5 layers of prismatic cells in all cases. The mesh sizes were approximately $350,000,550,000$, and 800,000 elements for patients 1,2, and 3 , respectively. The maximum element size was approximately $0.3 \mathrm{~mm}$ in all cases. Flow was then simulated in the resulting finitevolume model assuming the circulating fluid to be Newtonian and incompressible with an attenuation of $1050 \mathrm{~kg} / \mathrm{m}^{3}$ and a viscosity of $0.003 \mathrm{~kg} / \mathrm{m} / \mathrm{s}$. Vessels were considered to be rigid tubes. Flow was simulated by using the CFX 11.0 commercial software (ANSYS). The analytic cardiac cycle was 0.8 seconds with a time-step of $0.01 \mathrm{sec}-$ onds, so that 1 cycle comprised 80 time-steps.

We simulated a total of 3 cycles, making sure that the initial transients disappeared. Flow was considered pulsatile with a synthetic inlet velocity function reproducing a realistic shape of the cardiac function. The mean inlet velocity varies between 1 and $0.37 \mathrm{~m} / \mathrm{s}$ within 1 cardiac cycle with a parabolic distribution in space. Constant pressure was used as the outlet boundary condition, preceded by an additional artificial resistance. The resistance was modeled as a porous layer on the basis of Darcy's law by taking only the part of the pressure loss into account that is linearly proportional with the velocity. The parameters of the outlet boundary were set on the basis of the nature of the peripheral resistance and the almost constant pressure at the level of capillaries. The parameters were chosen so that the fluctuation of the average pressure level in the region under investigation remained between 80 and $120 \mathrm{~mm} \mathrm{Hg}$. This method leads to a velocity and pressure field inside the studied vessel segments fluctuating in the same phase.

Local flow fields of the affected vascular segment, namely of the basilar bifurcation (patient 1), the superior wall of the $\mathrm{C} 2$ segment of the ICA (patient 2), and of the PcomA origin (patient 3) were determined by CFD analysis. CFD gave the velocity field, surface distributions of relative surface pressure on the wall, luminal WSS, and SWSSG. Comparing the initial and the follow-up 3D-DSA images, we defined the vascular segment of interest in which the aneurysm developed. At this segment in alignment with blood flow, a virtual line was drawn, along which WSS and SWSSG was measured. The local flow fields were also determined on 2 models with the developed aneurysms, in which a 3D-DSA was performed after the formation of the aneurysm. The third patient, with an aneurysm at the basilar bifurcation, refused checkup angiograms, and she was followed with MR angiography, so a 3D-DSA for CFD modeling was not available in this case.

\section{Results}

\section{Patients and Hemodynamic Microenvironment}

Case 1. A 52-year-old woman with a history of severe obesity and hypertension was admitted with a ruptured 6-mm basilar tip aneurysm. During the hospital stay, the aneurysm endogenously thrombosed and demonstrated healing and complete remodeling of the basilar bifurcation, as previously described. ${ }^{13}$ Because the aneurysm did not show any signs of recurrence until 20 months, we assume that endogenous thrombus formation was subsequently followed by shrinkage of the aneurysm and remodeling of the basilar bifurcation. On the 20-month follow-up MR imaging and MRA, a small 3-mm regrown aneurysm was observed at the level of the former aneurysm neck, which remained unchanged during the following 5 years (Fig 1). A 3D-DSA for CFD simulations was performed at the 3-month follow-up, when the basilar apex showed a complete remodeling, without an aneurysm remnant.

At the basilar apex, the surface pressure was relatively high compared with neighboring areas (Fig $1 B$ ). The numeric values of the pressure are not given because as a characteristic of incompressible flow with rigid walls, only the pressure gradients are relevant in the formation of the flow. The same flow field would result with an arbitrary constant added to the pressure field. The temporal average of the WSS in the parent vessel was $7 \mathrm{~Pa}$. The WSS detected along the predefined line showed extremely high values, reaching nearly $40 \mathrm{~Pa}$ at peak systole, almost 6 times the average value. The WSS at its peak was immediately preceded by a short approximately 1-mmlong area, where the SWSSG showed a positive peak, exceeding $40 \mathrm{~Pa} / \mathrm{mm}$ (Fig 1C). The proximal edge of the later-forming aneurysm neck matched this area under high hemodynamic stress (Fig 1D).

Case 2. A 44-year-old female patient, with a history of smoking, was treated due to a ruptured AcomA aneurysm and an unruptured basilar bifurcation aneurysm. Twenty-five months later, she developed an 8-mm aneurysm at the upper wall of the C2 segment of the right ICA (Fig 2). The 3D-DSA for CFD simulations was obtained at the time of the AcomA aneurysm rupture, when no aneurysm was present at the C2 segment, and also 25 months later, with the already-developed aneurysm.

The surface pressure at the area of interest was relatively increased (Fig 2B). The temporal average of the WSS in the parent vessel was 9.5 $\mathrm{Pa}$. The WSS measured along the predefined line showed an oscillating pattern, with a peak of $>60$ $\mathrm{Pa}$ at the 5-mm monitoring point (approximately 6 times the average value), a location that corresponded to the proximal edge of the future aneurysm neck along this line. The WSS peak was preceded by an increased positive SWSSG at $3 \mathrm{~mm}$, reaching nearly $40 \mathrm{~Pa} / \mathrm{mm}$. The SWSSG also showed an undu- 

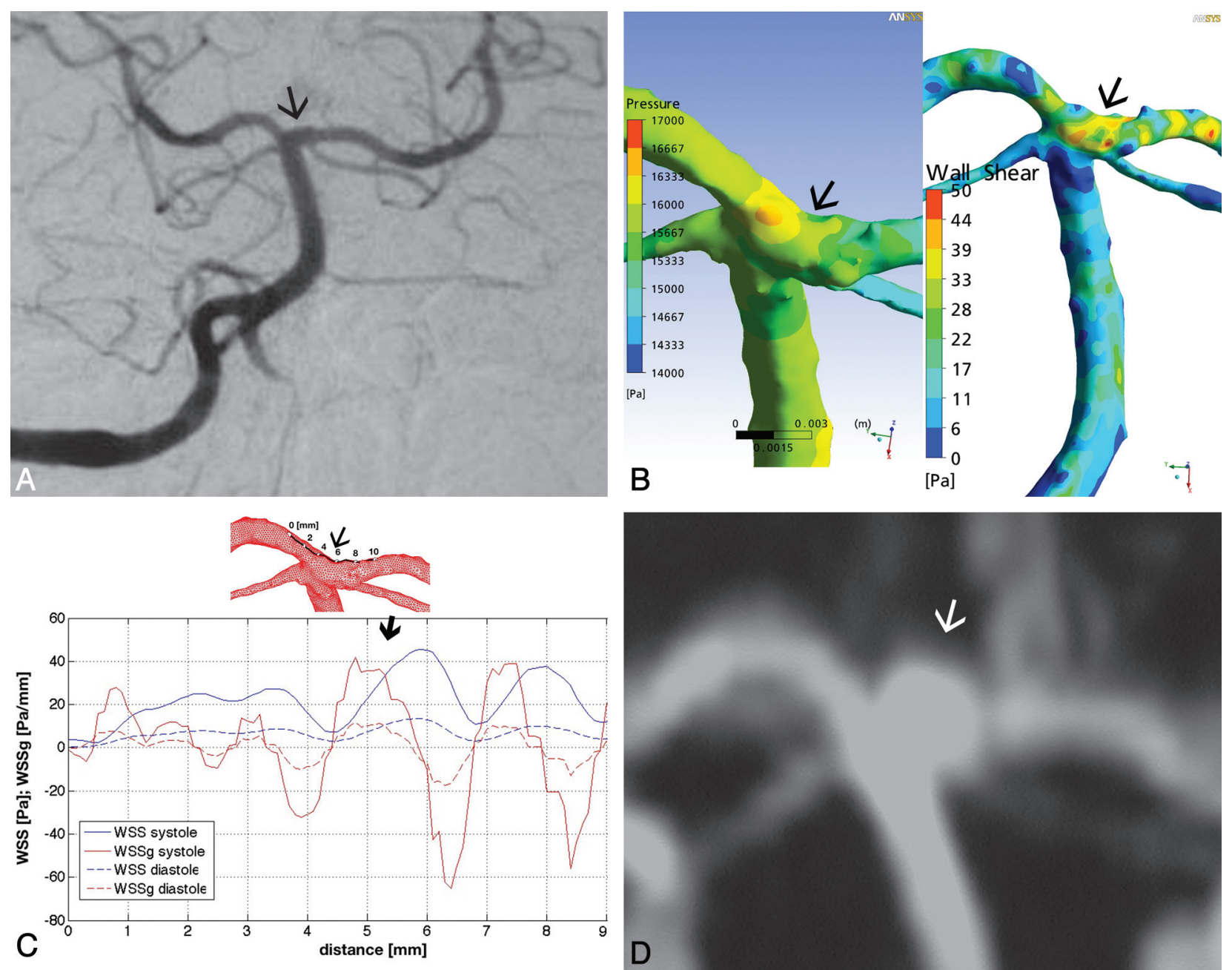

Fig 1. A, Basilar artery. Posteroanterior Townes view DSA of patient 1 shows no aneurysm at the basilar bifurcation. $B$, Surface pressure (left) and WSS distribution map (right) demonstrate significant forces attacking the basilar tip. C. Along the arbitrarily defined line, the diagram shows a major peak in SWSSG at point 5 (thin arrow), followed immediately by a WSS peak (thick arrow). $D$, This point corresponds to the proximal edge of the later developed aneurysm neck as shown by the time-of-flight MRA (arrow indicates the future aneurysm site on $A$, $B, C$, and the developed aneurysm on $D$ ).

lating pattern, with a second more significant peak at approximately $7 \mathrm{~mm}$, reaching $>60 \mathrm{~Pa} / \mathrm{mm}$, which was also accompanied by an increased, though smaller, WSS peak compared with the previous one. These latter peaks of WSS and SWSSG fell into the future neck area (Fig 2C, $-D$ ).

The fluid dynamic analysis of the model with the developed aneurysm showed a relatively uniform surface pressure distribution on the aneurysm wall, which was lower compared with the proximal parent artery (Fig 3A). The WSS was elevated at the edges, more significantly at the proximal and distal edges of the aneurysm neck (Fig 3B). The WSS map showed amplitude increases along the inflow and outflow regions throughout the cardiac cycle but without temporal and spatial oscillations. The flow field demonstrated 1 dominating vortex inside the aneurysm, which remained stable throughout the cardiac cycle, and showed no definable impingement region (Fig 3C).

Case 3. A 58-year-old female patient was first treated for an incidentally discovered right $4-\mathrm{mm}$ carotid cave aneurysm. At the time of the treatment, a tiny aneurysm-like dilation was observed at the origin of the right PcomA. Twenty-three months later the patient had a subarachnoid hemorrhage from the PcomA aneurysm, which had grown to an 8-mm size (Fig
4). The 3D-DSA used for CFD simulations was obtained at the time of the carotid cave aneurysm treatment, when only a tiny aneurysm beginning was present at the PcomA origin.

Given the relatively large size of the PcomA in this case, the branching point of the PcomA was arbitrarily considered as a true vascular bifurcation, where flow is divided into the 2 arising branches. The line of measurements was drawn at the upper wall of this bifurcation, crossing over the sac of the already present small aneurysm beginning (Fig 4D). The surface pressure distribution was relatively increased in this area and more or less evenly distributed on the upper wall of the bifurcation, including the distal wall of the aneurysm beginning. The temporal average WSS of the parent vessel was $14 \mathrm{~Pa}$ (Fig. 4C). The pattern of the WSS along the line of measurement showed 2 peaks: a smaller one at $3.5 \mathrm{~mm}$ of approximately $50 \mathrm{~Pa}$ and a bigger one at $7 \mathrm{~mm}$, reaching $>80 \mathrm{~Pa}$ (ie, $>5$ times the average value). Both peaks corresponded to the edges of the aneurysm beginning. These WSS peaks were preceded by an extremely high SWSSG peak of approximately $90 \mathrm{~Pa} / \mathrm{mm}$. The point where both measures were extremely high was $7 \mathrm{~mm}$, which corresponded to the anatomically distal but, according to the flow direction, proximal aneurysm neck (the flow was divided 

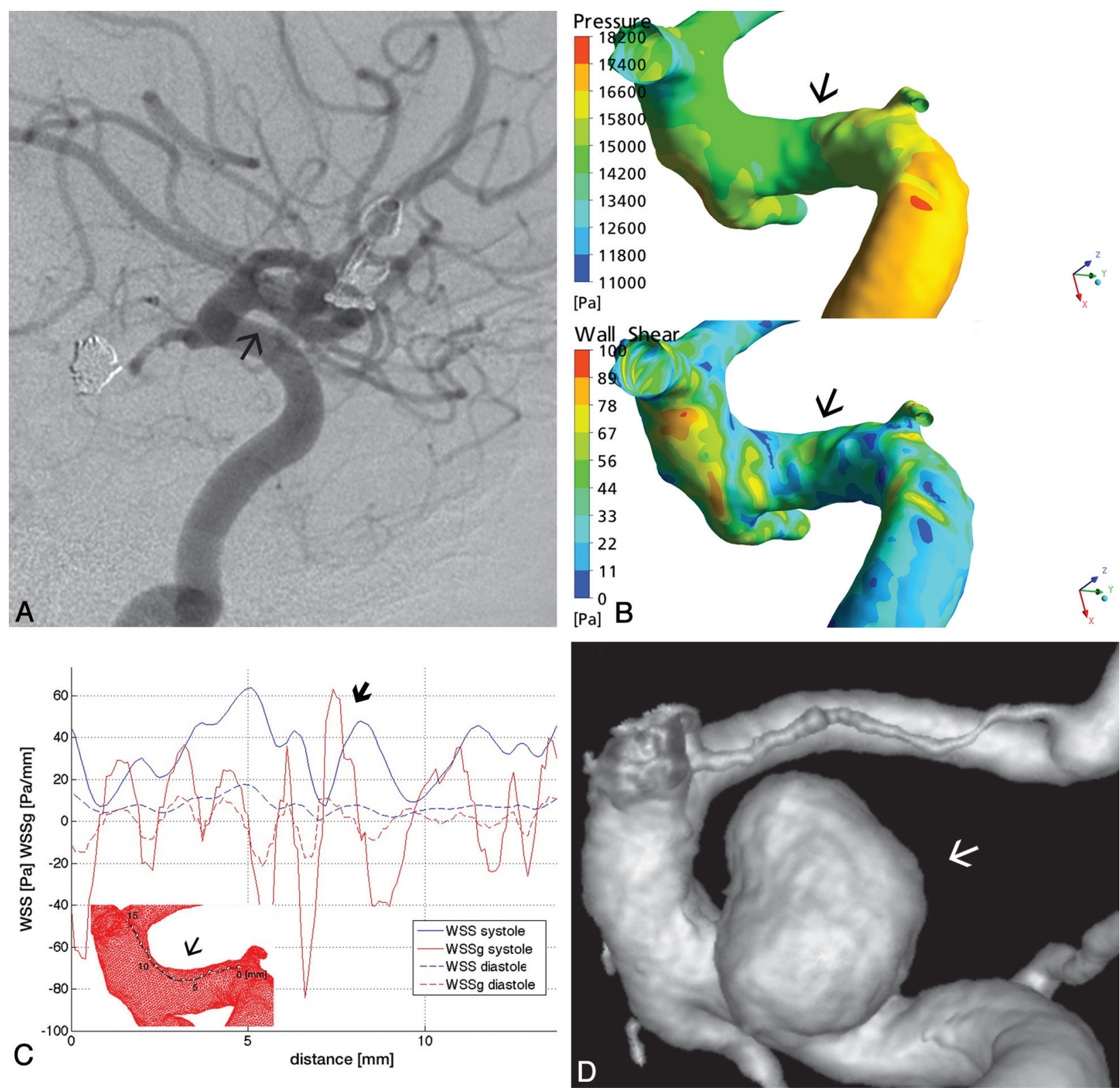

Fig 2. A, Right ICA lateral view DSA of patient 2 shows no aneurysm at the upper wall of the C2 ICA segment (arrow). $B$, Surface pressure (upper) and WSS distribution map (lower) demonstrate increased forces at the site of future aneurysm. C, Along the arbitrarily defined line, the diagram shows a major peak in SWSSG approximately at point 3 (thin arrow), followed immediately by a WSS peak at point 5 (thick arrow). $D$, The first combined peak is followed by a second at approximately points $7-8$, which fell later into the neck area of the developed aneurysm, as seen on the 3D reconstructed angiogram.

at this point for the distal carotid artery and the PcomA) (Fig $4 D,-E)$.

The flow dynamic analysis of the developed and ruptured aneurysm showed an evenly distributed elevated pressure at the aneurysm surface (Fig 5A). The WSS map showed an elevated WSS at the proximal medial and distal edge of the aneurysm neck and an oscillating pattern in the aneurysm dome throughout the cardiac cycle, with increasing values at peak systole (Fig 5B). Studying the flow field, we found a highly unsteady flow region near the aneurysm neck. The velocity distribution inside the aneurysm demonstrated a relatively small inflow jet, a changing small impingement region, and multiple changing intra-aneurysmal vortices (Fig 5C).

\section{Discussion}

By observing the natural history of aneurysm formation from its preaneurysm state and by using $3 \mathrm{D}$ reconstructions of the vascular segments not yet harboring a true aneurysm, we have performed CFD analysis at both sidewall and bifurcation arterial segments where aneurysms later developed. Increased WSS accompanied by high positive SWSSG was found at the sites of future aneurysms, potential factors involved in aneurysm initiation.

More and more evidence indicates that hemodynamics play a role in the life cycle of cerebral aneurysms. CFD studies have demonstrated major hemodynamic forces affecting arterial bifurcations, branching points, and curvatures of the circle of Willis, including constantly high surface pressure and 

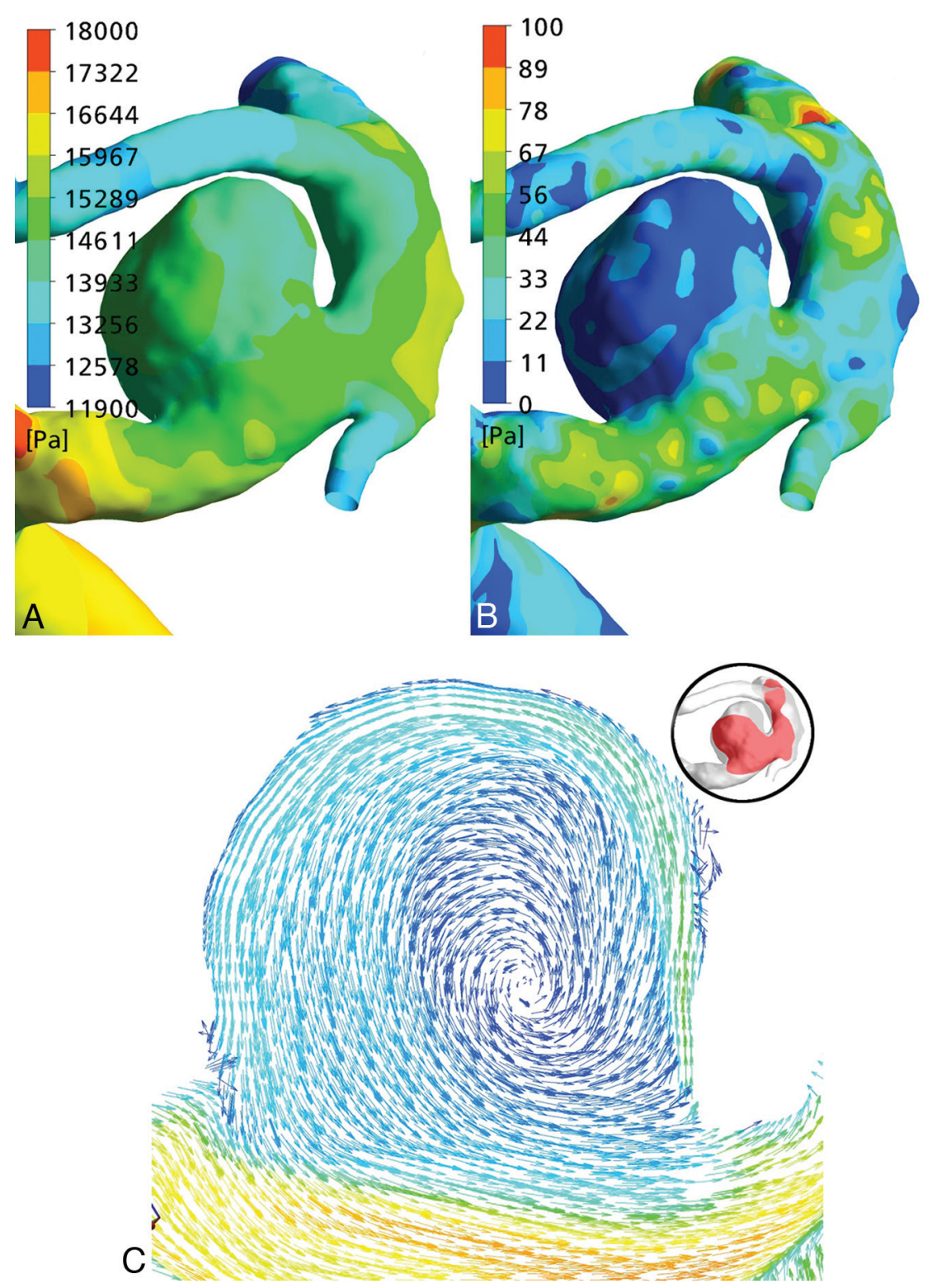

Fig 3. $A$ and $B$, Surface pressure $(A)$ and WSS $(B)$ distribution maps of the aneurysm of patient 2. $C$, Velocity vectors of the same aneurysm show a simple flow pattern without a well definable impingement zone.

WSS. ${ }^{1,2}$ Both of these measures are significantly influenced by the vessel geometry and, thus, may show significant interindividual differences. ${ }^{1}$ The seeming relationship between elevated stresses and the preferential locations of cerebral aneurysm was further strengthened by research on the biologic response of the vessel wall to these mechanical stimuli. It has been suggested that normal functioning of the endothelial cells is mediated through WSS ${ }^{5,14}$ and that high WSS is linked to different biologic reactions, such as increased metalloproteinase ${ }^{15}$ and nitric oxide production, ${ }^{16,17}$ which may play a role in pathologic vessel wall remodeling and aneurysm formation.

Although intra-aneurysmal hemodynamics has been quite extensively studied and linked to aneurysm growth and rupture risk, ${ }^{18-21}$ much less is known about the specific hemodynamic microenvironment, which may initiate aneurysm formation. Besides the incriminated high WSS, effort has been made to identify other hemodynamic measures that may play a role in this process, like the oscillatory shear index, ${ }^{22}$ the GON,${ }^{10}$ and the AFI. ${ }^{9}$ In animal models of artificially created bifurcations, hemodynamics was related to biologic reaction and concomitant vessel wall remodeling, as proved by histology. ${ }^{7,8}$ Wang et al associated the combination of increased WSS and high positive SWSSG with specific molecular alterations in the vessel wall, which were formerly implicated in aneurysm formation. ${ }^{17}$ They speculated that a high positive SWSSG may compromise the normal physiologic response of the endothelial cells to high WSS. The stretching forces exerted by the shear stress gradient would loosen the intercellular contact between the endothelial cells, leading to the onset of destructive remodeling.

The setting of combined high WSS and SWSSG, as a valid measure of aneurysm initiation, could not be confirmed in a computational study on human vessels, where flow simula- 

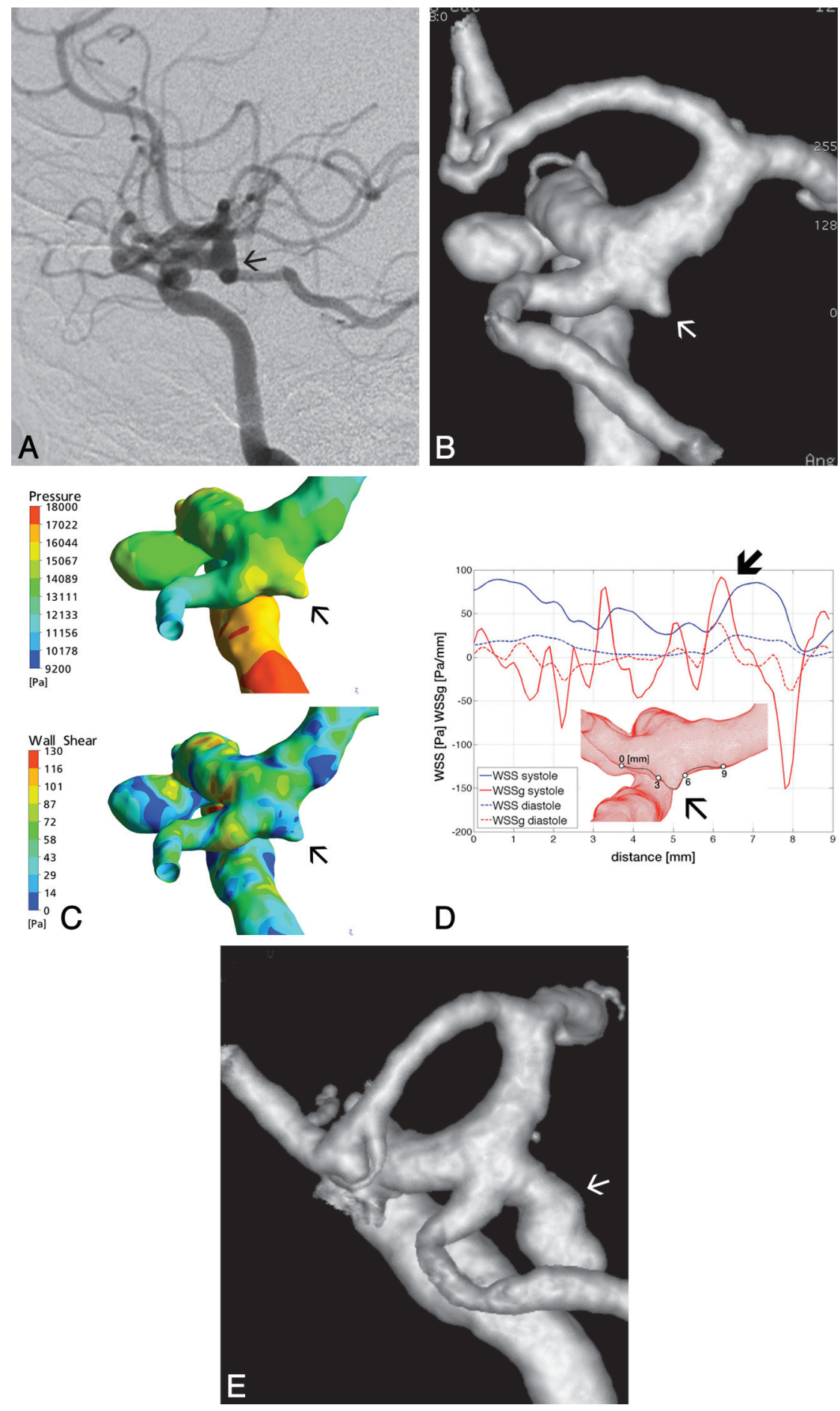

Fig 4. $A$ and $B$, Lateral view DSA of the right ICA of patient 3 shows an aneurysm at the carotid cave; however, no aneurysm beginning is visible at the origin of the PcomA (A), demonstrated by the $3 \mathrm{D}$ reconstruction angiogram (arrows, $B$ ). $C$, Surface pressure (upper) and WSS distribution map (lower) demonstrate increased forces at the edges of the tiny aneurysm beginning (arrows). D, Along the arbitrarily defined line, the diagram shows a first major peak in SWSSG approximately after point 3, followed immediately by a lower WSS peak and again at points 6 and 7 respectively (thick arrow). E, These points correspond to the proximal and distal edges of the aneurysm beginning, which further evolved to a larger aneurysm that ruptured (arrow).

tions were performed on vessels harboring a sidewall aneurysm, after artificial reconstruction of the parent vessel by computational methods. ${ }^{10}$ Shimogonya et al ${ }^{10}$ have suggested that the fluctuation of the SWSSG over 1 pulse cycle, as measured by the GON, may have a better prognostic value. These results are not necessarily contradictory to our findings, because we could also show an important amplitude difference in the systolic and diastolic SWSSG in all 3 cases (Figs $1 C, 2 C$, and $4 D$ ), also suggesting the temporal fluctuation of the SWSSG over the cardiac cycle. On the other hand, the reason for this apparent discrepancy might be related to the fact that the hemodynamic environment may differ at a bifurcation and at a sidewall location. Second, the artificially recreated vascular segment in the study by Shimogonya et $\mathrm{al}^{10}$ may not perfectly reflect the baseline vascular geometry, because a growing aneurysm, by its mass effect, may distort the original 

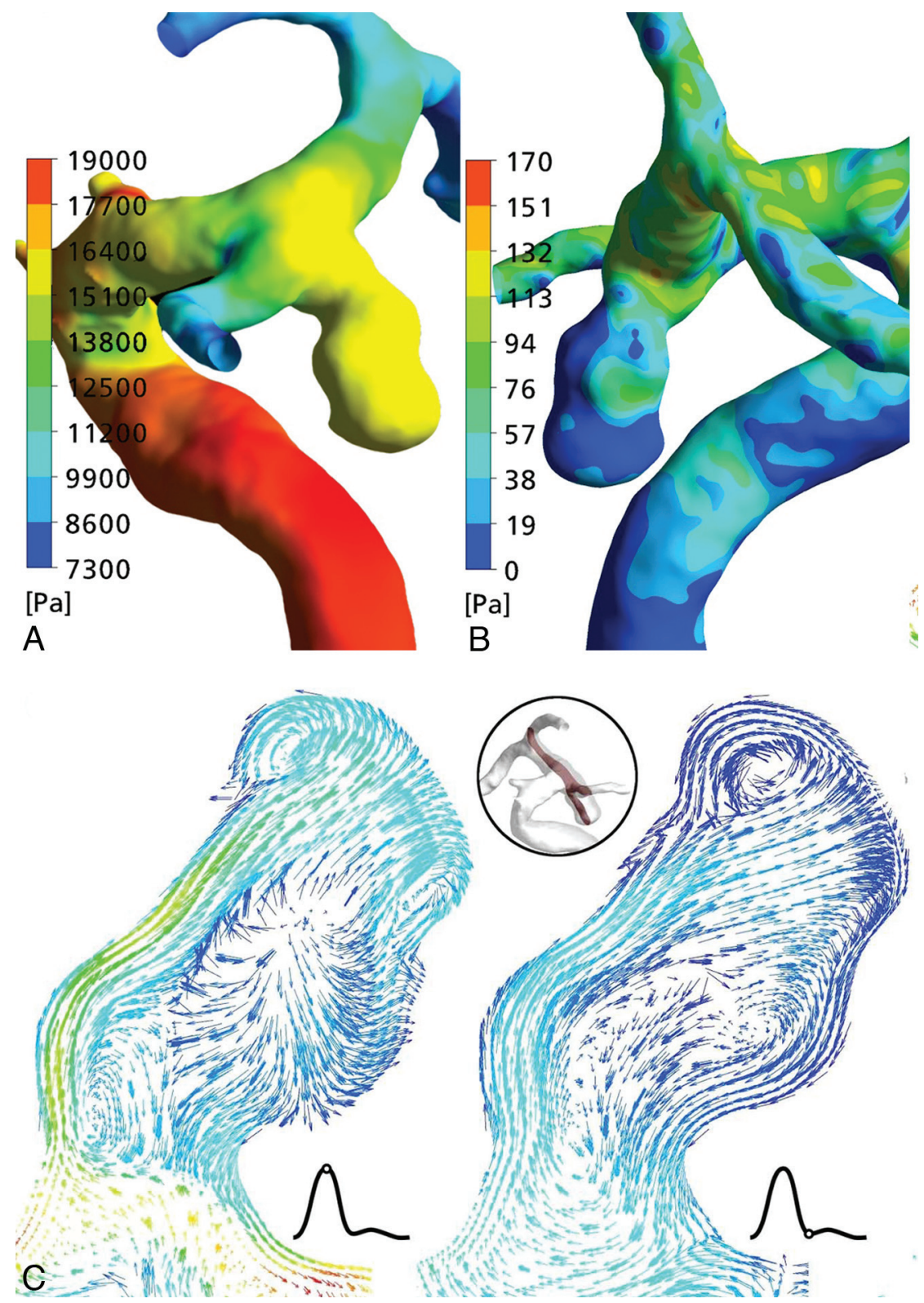

Fig 5. $A$ and $B$, Surface pressure $(A)$ and WSS distribution $(B)$ on the aneurysm wall of patient 3. $C$, The velocity vector fields show a changing impingement zone at the aneurysm dome throughout the cardiac cycle and changing multiple vortices in the aneurysm lumen.

vascular anatomy. Changing geometry has a very important impact on flow fields, ${ }^{1}$ meaning the retrospective reconstruction of a parent vessel may not necessarily show the same flow patterns as at baseline. On the basis of these principles, in our study, we focused on the analysis of WSS and SWSSG, and our cases could reflect both sidewall and bifurcation locations.

The relative surface pressure at the affected segments was increased in all 3 patients. In those 2 patients in whom originally no aneurysm was present, the high peak in WSS and the SWSSG peak presented exactly at the site of the proximal edge of the neck of the aneurysm that developed later. The WSS values at these points were at least 5 times higher than the temporal average values of the parent vessel. One can assume that the initial destructive changes in the vessel wall, like rupture of the internal elastic lamina and weakening of the muscular layer, take place at the site of the highest hemodynamic stress. However, the combination and the relative magnitudes of WSS and SWSSG at similar vascular segments of healthy individuals not developing an aneurysm need to be investigated. In patient 1 , the aneurysm developed at the site of the bifurcation where both of these parameters were increased, whereas on the other side of the bifurcation, both parameters were closer to normal, a condition that may also reflect the importance of individual vascular anatomy.

Once an aneurysm beginning is formed, as in patient 3 , the hemodynamic microenvironment will change. The major stresses will be shifted to attack a broader area of the aneurysm neck, as shown by the simulation on the models with developed aneurysms. These forces, together with low WSS on the aneurysm wall, may lead to the growth of the aneurysm sac and neck. ${ }^{20,21}$ The hemodynamic analysis of the 2 developed aneurysms in our patients reflected previously published data. ${ }^{18}$ The flow analysis of the ruptured PcomA aneurysm of patient 3 showed a small inflow jet with changing impinge- 
ment zones and multiple intra-aneurysmal vortices, described as type IV pattern by Cebral et al. ${ }^{18}$ This flow pattern was described as most often present in PcomA aneurysms and was also correlated with elevated risk of rupture. ${ }^{18}$ On the other hand, the nonruptured aneurysm of patient 2 demonstrated a stable 1-vortex flow pattern, without a definable impingement zone. This type I flow pattern, defined as the simplest, was specific for unruptured aneurysms. ${ }^{18}$

The potential significance of our observations may be limited by the low number of patients studied; by the fact that at baseline, 2 of the 3 studied arterial segments (patients 1 and 3 ) may not be considered as completely healthy at a tissue level; and by the lack of similar measurements on healthy controls not developing an aneurysm.

\section{Conclusions}

By observing the natural history of aneurysm formation from its preaneurysm state, in correspondence with previous animal experimental data, the current CFD study with clinical follow-up suggests that the combined presence of increased WSS and high positive SWSSG focused at a small arterial segment may have a role in the initiation process of an aneurysm. Because both of these parameters are relatively easily deducible by CFD simulations, they may be considered for further analysis in this disease process. The exact pathomechanism of how this complex hemodynamic setting will translate to a biologic response also needs further study.

\section{References}

1. Alnaes MS, Isaksen J, Mardal KA, et al. Computation of hemodynamics in the circle of Willis. Stroke 2007;38:2500-05

2. Takeuchi S, Karino T. Flow patterns and distributions of fluid velocity and wall shear stress in the human internal carotid and middle cerebral arteries. Surg Neurol 2009 Oct 22 [Epub ahead of print]

3. Foutrakis GN, Yonas H, Sclabassi RJ. Saccular aneurysm formation in curved and bifurcating arteries. AJNR Am J Neuroradiol 1999;20:1309-17

4. Schirmer CM, Malek AM. Wall shear stress gradient analysis within an idealized stenosis using non-Newtonian flow. Neurosurgery 2007;61:853-63, discussion 863-64
5. Malek AM, Alper SL, Izumo S. Hemodynamic shear stress and its role in atherosclerosis. JAMA 1999;282:2035-42

6. Resnick N, Yahav H, Shay-Salit A, et al. Fluid shear stress and the vascular endothelium: for better and for worse. Prog Biophys Mol Biol 2003;81:177-99

7. Meng H, Swartz DD, Wang Z, et al. A model system for mapping vascular responses to complex hemodynamics at arterial bifurcations in vivo. Neurosurgery 2006;59:1094-100, discussion 1100-01

8. Meng H, Wang Z, Hoi Y, et al. Complex hemodynamics at the apex of an arterial bifurcation induces vascular remodeling resembling cerebral aneurysm initiation. Stroke 2007;38:1924-31

9. Mantha A, Karmonik C, Benndorf G, et al. Hemodynamics in a cerebral artery before and after the formation of an aneurysm. AJNR Am J Neuroradiol 2006;27:1113-18

10. Shimogonya $\mathrm{Y}$, Ishikawa $\mathrm{T}$, Imai $\mathrm{Y}$, et al. Can temporal fluctuation in spatial wall shear stress gradient initiate a cerebral aneurysm? A proposed novel hemodynamic index, the gradient oscillatory number (GON). $J$ Biomech 2009;42:550-54

11. Szikora I, Paal G, Ugron A, et al. Impact of aneurysmal geometry on intraaneurysmal flow: a computerized flow simulation study. Neuroradiology 2008;50:411-21

12. Paal G, Ugron A, Szikora I, et al. Flow in simplified and real models of intracranial aneurysms. International Journal of Heat and Fluid Flow 2007;28:653-64

13. Kulcsar Z, Berentei Z, Marosfoi M, et al. Thromboembolic complication induced stable occlusion of a ruptured basilar tip aneurysm: case report and review of the literature. Interv Neuroradiol 2010;16:83-88

14. Resnick N, Collins T, Atkinson W, et al. Platelet-derived growth factor B chain promoter contains a cis-acting fluid shear-stress-responsive element. Proc Natl Acad Sci U S A 1993;90:7908

15. Sho E, Sho M, Singh TM, et al. Arterial enlargement in response to high flow requires early expression of matrix metalloproteinases to degrade extracellular matrix. Exp Mol Pathol 2002;73:142-53

16. Fukuda S, Hashimoto N, Naritomi $\mathrm{H}$, et al. Prevention of rat cerebral aneurysm formation by inhibition of nitric oxide synthase. Circulation 2000;101:2532-38

17. Wang Z, Kolega J, Hoi Y, et al. Molecular alterations associated with aneurysmal remodeling are localized in the high hemodynamic stress region of a created carotid bifurcation. Neurosurgery 2009;65:169-77, discussion 177-78

18. Cebral JR, Castro MA, Burgess JE, et al. Characterization of cerebral aneurysms for assessing risk of rupture by using patient-specific computational hemodynamics models. AJNR Am J Neuroradiol 2005;26:2550-59

19. Cebral JR, Sheridan M, Putman CM. Hemodynamics and bleb formation in intracranial aneurysms. AJNR Am J Neuroradiol 2010;31:304-10

20. Boussel L, Rayz V, McCulloch C, et al. Aneurysm growth occurs at region of low wall shear stress: patient-specific correlation of hemodynamics and growth in a longitudinal study. Stroke 2008;39:2997-3002. Epub 2008 Aug 7

21. Jou LD, Lee DH, Morsi $\mathrm{H}$, et al. Wall shear stress on ruptured and unruptured intracranial aneurysms at the internal carotid artery. AJNR Am J Neuroradiol 2008;29:1761-67

22. Singh PK, Marzo A, Howard B, et al. Effects of smoking and hypertension on wall shear stress and oscillatory shear index at the site of intracranial aneurysm formation. Clin Neurol Neurosurg 2010;112:306-13. Epub 2010 Jan 21 